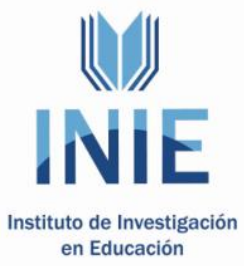

Actualidades Investigativas en Educación

Revista Electrónica publicada por el

Instituto de Investigación en Educación

Universidad de Costa Rica

ISSN 1409-4703

http://revista.inie.ucr.ac.cr

COSTA RICA

CONFERENCIAS EN EL MARCO DE LA NEGOCIACIÓN DEL FONDO ESPECIAL PARA LA EDUCACIÓN SUPERIOR (FEES)

Volumen 10, Número Especial dedicado al SOCIEDAD, DESARROLLO Y UNIVERSIDAD PÚBLICA

Dr. José Narro Robles

Rector de la Universidad Nacional Autónoma de México (UNAM)

ALGUNOS RETOS DE LA UNIVERSIDAD PÚBLICA

EN AMÉRICA LATINA

\author{
Dra. Yamileth González García \\ Rectora de la Universidad de Costa Rica (UCR) \\ VÍNCULO UNIVERSIDAD-SOCIEDAD, VITAL PARA EL DESARROLLO HUMANO \\ SOSTENIBLE
}

Este número se publicó el 15 de noviembre de 2010

La revista está indexada en los directorios:

LATINDEX, REDALYC, IRESIE, CLASE, DIALNET, DOAJ, E-REVIST@S,

La revista está incluida en los sitios:

REDIE, RINACE, OEI, MAESTROTECA, PREAL, HUASCARAN, CLASCO

Los contenidos de este artículo están bajo una licencia Creative Commons 


\section{ALGUNOS RETOS DE LA UNIVERSIDAD PÚBLICA EN AMÉRICA LATINA}

\section{Dr. José Narro Robles ${ }^{1}$ \\ Rector \\ Universidad Nacional Autónoma de México (UNAM)}

Saludo a la Dra. Yamileth González García, Rectora de la Universidad de Costa Rica. Agradezco en todo lo que vale la invitación que me formuló para asistir de nueva cuenta a platicar con alumnos y maestros de esta Universidad.

Gracias por su presentación. El secreto de la vida es tener buenos amigos. Estimadas vicerrectoras y vicerrectores; decanas y decanos, académicos y alumnos; colegas de México, doctoras Norma Blazques y Olga Bustos, señores y señoras.

En esta ocasión revisaré algunos de los principales retos que enfrentan las universidades públicas de América Latina. Por supuesto, no son todos y no los tocaré con la profundidad con que se deben considerar.

Quiero iniciar la presentación con una expresión de absoluta convicción: la universidad, como institución social desde su establecimiento en 1088 en Bolonia, Italia, ha desempeñado un papel trascendente. Para entender mejor su función en la sociedad, y su responsabilidad ante ella, es necesario tener conciencia tanto de su pasado como de un futuro probable, ya que ambos influyen en su presente. Lo que hoy hacemos es viable por lo que se hizo y se realiza para fundamentar lo que se hará en el porvenir.

La universidad moderna forma parte del sistema educativo como su componente más elevado, al igual que del sistema de ciencia que encuentra en ella un espacio privilegiado para el desarrollo de la investigación. Así, en la universidad actual confluyen dos tipos de racionalidad, la educativa y la científica, regidas por lógicas distintas, pero ligadas íntimamente al conocimiento. La primera implica la transmisión sistemática del saber adquirido. En tanto que para la segunda, el propósito fundamental radica en la producción de conocimiento nuevo.

\section{EL MUNDO DE HOY}

Para apreciar la dimensión de los retos y compromisos que tiene la universidad es

1 Para más información consultar el sitio http://www.dgi.unam.mx/rector/

Volumen 10, Número Especial, Año 2010, ISSN 1409-4703 
preciso tener una idea, así sea general, de algunas condiciones del mundo en que vivimos. Este se caracteriza por la existencia de grandes paradojas. De una parte, se registran avances increíbles en la ciencia, la salud y la tecnología. De la otra, millones de personas viven en pobreza, son analfabetas, mueren de enfermedades para las que hay cura, o no tienen acceso real a los más elementales derechos y servicios.

El proceso de mundialización, producto en parte de la revolución científico tecnológica, sin duda ha redituado logros considerables en diversos ámbitos del desarrollo de la sociedad, cuya dimensión más claramente visible y preponderante es la económica. Sin embargo, los beneficios de esos avances y logros han sido desiguales para las naciones y grupos sociales. En este sentido, se puede afirmar que el mundo globalizado es, a la vez, sorprendente y contradictorio.

Algunos de los grandes logros de la humanidad están determinados por los niveles de educación y de acceso a la información; por el grado de desarrollo económico, de producción industrial y de comercio internacional; por el avance de la democracia en el mundo y la preocupación por los derechos humano; por la erradicación o el control de enfermedades décadas atrás diezmaban a la población, así como por el aumento en esperanza de vida.

No obstante, a la par de la mejoría persisten y se han agudizado viejos problemas y se han generado nuevas tensiones y rezagos. Se ha favorecido la concentración de la riqueza y se han incrementado la desigualdad y la exclusión social. La modernización tecnológica del proceso productivo ha generado desempleo y subempleo. Millones de personas, principalmente niños, mueren a causa de padecimientos de los que se conoce todo y para los cuales se dispone de las medidas preventivas y terapéuticas más efectivas. Se mantienen alta tasas de crecimiento poblacional en los países en desarrollo, y el deterioro ambiental ha alcanzado niveles tan graves que se ha puesto en riego no sólo la sustentabilidad del desarrollo, sino la vida en el planeta.

Estas contradicciones nos hacen ver lo necesaria que es la tarea de las universidades. Subrayan el compromiso que tenemos por delante de formar profesionales y ciudadanos con espíritu crítico y conciencia social; de seguir investigando en torno a nuestra sociedad y nuestra cultura, a través de las humanidades y las ciencias sociales, para entender y plantear soluciones a los problemas del presente y del futuro. Enfatizan la pertinencia de dar prioridad a la investigación científica sobre estos asuntos, sin menoscabo de la libertad de investigación, de mantener y ampliar nuestro vínculo con la sociedad a la que nos debemos como institución. En pocas palabras, enfrentamos la necesidad de llevar a efecto las 
funciones de docencia, investigación y extensión, con la calidad y pertinencia que nos demandan nuestros países y regiones.

Los retos que voy a comentar en la presentación tienen que ver, por un lado, con las tareas tradicionales de la universidad y, por el otro, con nuevos desafíos. En el primero de los casos me referiré a la transmisión y generación del conocimiento y a la extensión de sus beneficios de la forma más amplia posible, así como a la necesidad de mantener la autonomía de la universidad frente a cualquier tipo de poder: político, económico, religioso, civil, militar o de otra naturaleza. Entre los segundos me referiré al imperativo de la investigación, al grave problema del financiamiento y al compromiso con la sociedad.

\section{TRANSMISIÓN Y GENERACIÓN DE CONOCIMIENTO}

El conocimiento ha sido, históricamente, la clave en el desarrollo de la humanidad. Nuestra especie ha logrado avanzar en virtud de la razón y el saber sistematizado, igualmente, de la comprensión de lo que pasa en nuestros universos y de la posibilidad de entender, explicar y anticipar; de la capacidad para intervenir y modificar nuestros ambientes. El conocimiento ha hecho más libre al ser humano, pero también le ha permitido vivir en un mundo con mayor justicia y con más belleza. El conocimiento ha posibilitado al hombre desarrollarse con más comodidad, disminuir la incertidumbre, vencer la enfermedad, desarrollar sus potencialidades.

Con Karl Popper de la mano se puede sostener que el conocimiento humano representa un verdadero "milagro de nuestro universo". Sin embargo, esto no implica que se trate de un producto de la generación espontánea o del azar. Se trata del resultado de la preparación y del trabajo; de la observación y la reflexión; de la intuición. El método y la experimentación. Detrás del conocimiento hay otros saberes y mucha sistematicidad.

En la historia del planeta, la de nuestra especie es más bien breve. En la del ser humano, la del conocimiento es todavía más modesta. No han transcurrido más de 14 mil años desde que inició el proceso de domesticación de plantas y animales. Apenas hace siete mil quinientos años que se dieron en la India los primeros ejemplos de la escritura. Sólo han transcurrido cuarenta siglos desde la invención de la rueda o 32 desde el manejo del primer alfabeto. El dinero, ese oscuro invento que embelesa y corrompe, que resuelve problemas y genera otros de mayor gravedad, sólo existe en su dimensión de moneda de cambio desde hace dos mil 600 años.

Después del dinero, pero no como consecuencia del mismo, nacen la filosofía y la Volumen 10, Número Especial, Año 2010, ISSN 1409-4703 
ciencia y algunos años más tarde la democracia en Atenas. La carretilla, la brújula o el papel son jóvenes inventos de menos de dos mil años. No tenemos ninguna Universidad milenaria y la imprenta cumplirá en 31 años sus primeros seis siglos de existencia. Por increíble que parezca, hace apenas trescientos años el ser humano no había experimentado la revolución industrial ni tampoco la de los derechos del hombre. La clase media se concibió hace poco más de doscientos años y el telégrafo en su versión inicial no llega a esa edad. Para extrañeza de los jóvenes, las revoluciones del amor, de la moda y de la juventud no cumplen todavía 50 años y en 1980 no contábamos con celulares, Internet o computadoras personales.

El conocimiento siempre ha sido importante, pero en la actualidad es fundamental. Lo es para la economía de las naciones, pero más para el desarrollo de la sociedad. Es curioso, pero el conocimiento es indispensable para los individuos educados y estudiosos, pero todavía más para las mayorías que no tienen ese privilegio. No hay campo ni dimensión de la vida humana en la que no influye el saber en nuestros días.

Por ello, preocupa el desinterés de muchos países de la región en la materia. Por ello, angustia que el conocimiento, su producción y transmisión, no sean en muchas de nuestras naciones verdaderas prioridades. Es por esto que a muchos nos agobian la indiferencia en la realidad y el lugar común en el discurso. Sin educación superior, sin ciencia propia, una colectividad está condenada a la dependencia, a la medianía en el desarrollo, a viajar en el cabús del progreso.

La grave miopía política que nos afecta en muchos sitios, el increíble dogmatismo en torno a un modelo desvencijado que ya resulta contraproducente, al igual que la absurda subordinación a los modelos retro del exterior y el distanciamiento enfermizo respecto de los problemas y carencias de la mayoría, nos tienen sumidos en un pozo profundo y peligroso.

Y no se trata de un estado de ánimo afectado. Tampoco de una crisis del pesimismo que a muchos daña. Por desgracia, por desfortuna, no es así. Se trata de una visión realista de las cosas que resulta, entre otros, del análisis de los datos y de la comparación con otras regiones.

\section{AUTONOMÍA DE LAS UNIVERSIDADES}

La universidad, como una constante a lo largo de más de nueve siglos, puede definirse como un espacio de vida intelectual, de cultivo del conocimiento. Como una 
instancia o institución de naturaleza académica. El cultivo del saber forma parte de la función y razón de ser de la universidad, lo que implica preservar la cultura, enriquecerla y recrearla, transmitirla y difundirla.

Por esta misión y vocación, por el saber y la cultura, se distingue la naturaleza y especificidad de la institución universitaria en su inserción en el conjunto social y en el servicio singular que presta a la sociedad.

Sin embargo, la investigación, la docencia y la extensión pueden adoptar criterios y modalidades distintos, según las circunstancias y condiciones sociales e históricas y, por ello, la expresión de los fines y objetivos de las universidades puede ser diversa.

La tarea fundamental de cultivar el saber define entonces a la universidad. Como organización y espacio social, que tiene como eje de sustentación la cultura y, en alguna forma, lo que podría entenderse como la alta cultura, requiere de libertad como condición necesaria para realizarla; libertad de pensamiento, de búsqueda de la verdad y de expresión. Históricamente ha habido un reconocimiento de las diversas sociedades a esta tarea singular de la universidad.

La autonomía universitaria implica la fidelidad a la misión académica. La autonomía, inherente al quehacer académico, no significa una introversión y un repliegue de la universidad sobre sí misma que la incomunique del resto de la sociedad y la convierta en una "torre de marfil", sino al contrario: la actividad académica implica de manera esencial la comunicación del saber y de la cultura. Es en el desempeño de esa misión académica como la universidad interactúa con la sociedad, y es en ella donde procesa con sus propios criterios internos las demandas y requerimientos que la sociedad le hace.

La autonomía de la universidad ha alentado la existencia de los más valiosos principios educativos, como son: la libertad de cátedra e investigación, la pluralidad de posturas y creencias, éticas, políticas y religiosas, y de manera destacada la tolerancia y el respeto a la discrepancia.

Estos principios tienen una estrecha relación entre la autonomía institucional y la de las personas que integran la comunidad universitaria. El hecho de que una institución sea autónoma, en alguna forma, al menos deseable, implica que las personas que la constituyen también lo sean o que lleguen a serlo. En ese sentido, la autonomía es también un ideal que se persigue, especialmente, en la formación universitaria de los jóvenes, para que adquieran criterios propios de actuación en su vida adulta y profesional y tengan condiciones para que puedan valerse por sí mismos en forma responsable e independiente. En otro sentido, 
particularmente en las instancias colegiadas de deliberación y decisión, se requiere que las personas que toman decisiones en una institución autónoma sean ellas mismas autónomas (Dieterlen, 2004).

La autonomía y los mecanismos para su ejercicio han sido un dique de contención para ambiciones malsanas e intereses particulares, así como un antídoto para la ignorancia y la cerrazón. La autonomía no es un problema de las universidades públicas, sino una de sus principales fortalezas.

La autonomía permite a las universidades mantener la independencia necesaria frente al gobierno y otros poderes públicos, pero también frente a otros actores, grupos, partidos políticos, iglesias y organizaciones. Se ejerce respecto de todos ellos (Narro, 2004).

\section{GENERACIÓN DE NUEVO CONOCIMIENTO}

En la época actual, en la que emerge con fuerza el papel central del conocimiento tanto para la competitividad económica como para el desarrollo humano sustentable, es indiscutible la importancia de la investigación y del desarrollo de la ciencia y la tecnología en los países de nuestra región.

Es un hecho que por su historia y por el modelo institucional que se adoptó, de tipo profesionalizante, la gran mayoría de las universidades latinoamericanas prestaron atención tardía al desarrollo de la investigación, si se compara con algunas universidades estadounidenses que desde el siglo XIX siguieron el ejemplo de la reforma universitaria de Humboldt en Alemania y pusieron énfasis en la investigación, así como en la formación de investigadores.

También de manera tardía, en la mayoría de los países de América Latina los gobiernos comenzaron a prestar atención a la necesidad de establecer instancias estatales de impulso y apoyo al desarrollo de la ciencia y la tecnología.

En sentido estricto, a la fecha, pocas son las universidades latinoamericanas que pueden considerarse como verdaderas universidades de investigación. Aceptar esta situación no implica desconocer el esfuerzo que han hecho tanto algunas de las universidades como algunos de los gobiernos para incrementar las actividades en este sentido.

Entre los retos que afrontan la conformación y consolidación de la red iberoamericana de universidades de investigación recientemente establecida, la principal estriba en las Volumen 10, Número Especial, Año 2010, ISSN 1409-4703 
grandes asimetrías que existen entre las diversas regiones del mundo en cuanto a los desarrollos logrados y las capacidades que implican para la investigación. Estos logros y capacidades están concentrados sobre todo en una porción limitada del planeta.

Hebe Vessuri, en un publicación reciente: El rol de la investigación en la educación superior; implicaciones y desafíos para contribuir activamente en el desarrollo humano y social en el futuro, (GUNI, 2008) ilustra al respecto con los siguientes datos: más del $80 \%$ de los recursos destinados a la ciencia y a las universidades se gastaron en el área de la OCDE, y dentro de esta organización, la mayoría absoluta de las actividades han tenido lugar en América del Norte y Europa.

Unos pocos indicadores bastan para establecer la relación asimétrica: América del Norte y Europa juntos combinan el 95\% de los doctorados del mundo y siguen aventajando al resto de las naciones en la producción de nuevos doctorados a razón de 10 a 1. El 75\% del total de los artículos científicos proceden de América del Norte y Europa. Esta región concentra a la gran mayoría del profesorado universitario y, además, a la gran mayoría de las instituciones de alta calidad del mundo (Vessuri, 2008, p. 124).

Según el Observatorio Iberoamericano de la Ciencia, la Tecnología y la Sociedad, en el año 2007 el porcentaje de inversión en investigación y desarrollo por regiones era la siguiente: América del Norte 37.3 por ciento; Europa 31.9 por ciento; Asia 26.5 por ciento; América Latina y el Caribe 2.2 por ciento; Oceanía 1.9 por ciento y África 0.3 por ciento.

En América Latina y el Caribe en alguna forma también se replica esta asimetría, según la misma fuente el porcentaje de inversión en esta materia fue en 2007 la siguiente: Brasil 63.5 por ciento; México 17.7 por ciento; Argentina 5.8 por ciento; Chile 5.6 por ciento y el resto de América Latina y el Caribe 7.4 por ciento.

En materia de investigación hay un tema que me parece de especial trascendencia y que consiste en la tensión entre lo global y lo local. Considero que no se trata de un dilema en el que se deba optar por uno de los elementos, excluyendo al otro, sino más bien de mantener el equilibrio entre lo local y lo global. Esto requiere una mirada atenta a los avances mundiales de la ciencia y la tecnología y al mismo tiempo, una atención esmerada a las apremiantes necesidades y problemas de nuestras sociedades.

En este sentido, conviene resaltar que a la investigación se le presentan los retos y tensiones que la Comisión Internacional sobre la Educación para el Siglo XXI planteó en su Informe a la UNESCO en 1996: entre lo mundial y lo local, entre lo universal y lo singular, entre la tradición y la modernidad, entre el largo y el corto plazo, entre la indispensable 
competencia y la igualdad de oportunidades, entre el extraordinario desarrollo de conocimientos y la capacidad de asimilación, entre lo material y lo espiritual.

Con relación a esa última tensión, considero que hay que aceptar que el reciente énfasis mundial en la innovación, como componente esencial de la competitividad entre los países, nos puede conducir a descuidar la investigación en las humanidades y las ciencias sociales por considerarlas poco útiles y no redituables en la perspectiva económica, lo cual sería un grave error.

\section{FINANCIAMIENTO}

Por lo que toca al caso del financiamiento, no es mucho lo que puedo decir, pero lo señalo con toda convicción. En primer término, que un país que no destina los recursos necesarios a la educación superior, la ciencia y la cultura, es un país que está traicionando el porvenir de las próximas generaciones.

En segundo lugar, que el financiamiento que se otorgue no debe comprometer la libertad de cátedra y de investigación, ni la organización y el gobierno de la universidad. En adición hay que señalar que la universidad debe hacer un uso escrupuloso de los recursos, tener transparencia en su aplicación y rendir cuentas a la sociedad.

Igualmente, es necesario que los universitarios procuren allegarse recursos que ellos y su trabajo generen. La prestación de servicios y las alianzas con las entidades públicas y privadas del sector productivo son una fuente importante de recursos.

Finalmente, se debe entender que las universidades requieren certidumbre, por lo que la existencia de presupuestos multianuales es un imperativo en la actualidad. Es imposible planificar y operar pertinentemente una universidad, cuando los esfuerzos se concentran en la obtención de recursos para cubrir lo indispensable.

\section{COMPROMISO SOCIAL}

En la actualidad está plenamente reconocido que las universidades, sobre todo las de carácter público, tienen una función social y una responsabilidad con la colectividad. Sus funciones sustantivas no son fines en sí mismo y sí constituyen medios para responder a la sociedad. El servicio a esta última, en la óptica del desarrollo humano sustentable, es un propósito que debe impregnar y dar sentido a las actividades académicas de las universidades públicas.

La perspectiva de un futuro idéntico no debe abarcar a todas las universidades por Volumen 10, Número Especial, Año 2010, ISSN 1409-4703 
igual. El discurso generalizante de un "deber ser" de las universidades públicas, en cuanto al desarrollo de sus funciones y la organización de sus actividades debe ser desafiando en cuanto a su sentido, su pertinencia y su eficacia. Es en cada una de las universidades y aún más, en cada dependencia universitaria, en donde se debe dar una reinterpretación de las demandas y requerimientos explícitos, desde la perspectiva del campo disciplinario de que se trate y de las posibilidades con que se cuente.

Es de destacar el énfasis que la UNESCO ha puesto en el compromiso de las universidades en este sentido. Conviene recordar la Declaración de 1998 que especifica que la educación superior debe reforzar sus funciones de servicio a la sociedad y más concretamente las actividades orientadas a la erradicación de la pobreza, de la intolerancia, de la violencia, del analfabetismo, del hambre, contra el deterioro del medio ambiente y en oposición a las enfermedades, principalmente a través de un enfoque inter y transdisciplinario para analizar los problemas y plantear las posible soluciones.

Una responsabilidad fundamental de las universidades públicas consiste en elevar y garantizar la calidad de sus actividades académicas. Los estudios universitarios, además, deben propiciar una mayor responsabilidad ciudadana y fomentar una mejor convivencia social, al igual que cultivar valores laicos que permitan superar prejuicios y fomentar una ética de responsabilidad, de solidaridad, de compromiso social.

Hay otros problemas y retos que no conviene soslayar. Entre ellos destaca la visión pragmática que predomina en el mundo de hoy, que le exige a la universidad resultados aplicables e inmediatos. Esta visión, vinculada con la mercantilización del conocimiento, relaciona indebidamente la actividad educativa con los criterios propios del mercado. A los que presionan a las universidades en este sentido, debemos recordarles que el trabajo de la academia y de la ciencia es arduo, laborioso, que requiere de tiempo para llegar a resultados y que debe tener un compromiso con la colectividad.

\section{CONCLUSIONES}

La universidad, como una constante a lo largo de nueve siglos, puede definirse como un espacio de vida intelectual, como una institución de naturaleza académica. El cultivo del saber formar parte de su función y razón de ser, lo que implica preservar la cultura, enriquecerla y recrearla, transmitirla y difundirla. Como organización y espacio social que tiene este eje de sustentación, requiere de libertad como condición necesaria para realizarla. 
La internacionalización de la educación superior cobra actualmente importancia fundamental, dado el desarrollo de las comunicaciones y el potencial de las nuevas tecnologías de la información. Resulta también fundamental si lo que se busca es reducir las asimetrías entre las regiones y los países del mundo. En esa perspectiva, y en la medida en que se han ido conformando bloques regionales por razones comerciales, pero también de identidades, resulta significativo el esfuerzo de los últimos años por configurar una verdadera red iberoamericana de cooperación y de intercambio académico. La presencia del Rector de la UNAM en esta prestigiada Universidad, es una muestra del interés que al respecto tenemos.

Por su parte, es en las universidades, sobre todo en las públicas, donde pueden interactuar, recrearse y transmitirse los valores que caracterizan a las sociedades democráticas. La solidaridad, la generosidad o el servicio han perdido terreno ante la competencia y el individualismo extremos. La acumulación de dinero y de bienes materiales se ha convertido en el símbolo del éxito. Las universidades tienen el compromiso de cultivar y transmitir los valores laicos que dan cohesión a las sociedades.

Como ya se señaló, algunas de las profesiones dedicadas al conocimiento, a la búsqueda de la verdad, las humanidades o las artes son consideradas como no productivas. Las universidades deben, con el apoyo de sus académicos, enfrentar la descalificación de estas disciplinas, reivindicarlas como esenciales para el desarrollo integral del país y del conocimiento mismo. El humanismo es una condición insustituible para el futuro de las universidades y para el de las sociedades.

Termino con un apunte acerca de los más grandes compromisos de la universidad: el de ayudar a construir una sociedad más justa. El conocimiento tiene una enorme trascendencia en la posibilidad de disminuir las abismales desigualdades que caracterizan a muchas de las regiones del mundo. Las universidades deben participar más activamente aportando conocimiento para la creación de nuevas alternativas de desarrollo, donde el ser humano y su bienestar, y no las variables macroeconómicas, sean lo verdaderamente importante.

Reitero mi agradecimiento a la señora Rectora de la Universidad de Costa Rica por su amable invitación y espero que podamos fortalecer la relación, el intercambio y la cooperación entre nuestras dos instituciones.

Muchas gracias. Volumen 10, Número Especial, Año 2010, ISSN 1409-4703 


\section{VÍNCULO UNIVERSIDAD-SOCIEDAD, VITAL PARA EL DESARROLLO HUMANO SOSTENIBLE}

\section{Dra. Yamileth González García Rectora Universidad de Costa Rica}

La excelente disertación del Dr. José Narro Robles ilumina nuevas y posibles rutas en la lucha que estamos dando por la obtención de mejores condiciones financieras para el Sistema Educativo Nacional Costarricense, y reafirma algunos de los principios que hemos estado defendiendo desde la Universidad de Costa Rica, como es el convencimiento de que el vínculo Universidad-Sociedad es vital para el Desarrollo Humano Sostenible.

En el caso específico de nuestro país permítame, doctor Narro, comentar que el rol de la UCR ha sido clave. Clave en el pasado, porque la universidad -la única en el país por treinta años- juega un papel fundamental al establecer los cimientos de un Estado Benefactor Redistributivo de la riqueza y lo juega hoy como parte de ese vínculo prioritario: desarrollo tecnológico, empresa y sociedad de la información y lo será mañana con los nuevos desafíos. Existe un eje histórico que atraviesa esta relación: la oportunidad y el posible acceso a una educación superior pública de alta calidad y de manera equitativa para los y las habitantes del territorio nacional.

Como universitarios y universitarias estamos comprometidas con el fortalecimiento de la educación pública en todos sus niveles y en todas las regiones del país, sobre todo, en aquellas que cuentan con mayores niveles de inequidad.

Todos nosotros sabemos que Costa Rica es un país que históricamente apuesta por darle prioridad al desarrollo de la educación; una nación que considera que la enseñanza es base del progreso, la mejor vía para la movilización social, la condición más importante de la democracia e incluso de la libertad, como lo afirmaban ya nuestros gobernantes a principios del siglo XIX.

El esfuerzo por desarrollar la educación desde los primeros años de nuestra vida independiente, le da al país rasgos singulares en el contexto latinoamericano. Cuando el Dr. José María Castro Madriz, inaugura la Universidad de Santo Tomás en 1844, lo hace con una perspectiva muy clara sobre la función que la enseñanza debe cumplir en la sociedad:

2 Para mayor información consultar el sitio http://www.rectoria.ucr.ac.cr/informe/index.php Volumen 10, Número Especial, Año 2010, ISSN 1409-4703 
plantea una comunión entre las letras y las carreras técnicas, considerando la educación como la base de los "buenos negocios" y la consecución del poder. El Dr. Castro cifra: "las expectativas de desarrollo, modernización, riqueza, poder persuasivo y paz en una organización del proceso educativo donde la Universidad está llamada a jugar el rol básico".

En la época actual, en la que se valora la globalización como espacio de intercambio de mercados y consumos, de comunicaciones al instante, de redes y efectos planetarios, la llamada sociedad del conocimiento sigue ponderándose como la vía más importante para lograr las transformaciones necesarias.

En la actual coyuntura de crisis económica mundial y, en el caso de Costa Rica, a las puertas de la definición del $V$ Convenio del Fondo Especial para la Educación Superior (FEES), se nos dice que la crisis es grave y que eso obliga a una restricción de recursos. Pero nosotros no podemos permitir -y no lo hacemos- que la coyuntura esparza una cortina de humo sobre nuestra visión de país desarrollado redistributivo y equitativo.

La educación ha sido y debe seguir siendo un derecho inalienable para los habitantes de nuestra nación, un derecho tan básico como la vivienda, el vestido o la salud. La educación como base para el desarrollo económico, social, libre y democrático de un país debe ser considerada un bien social y no un medio de obtener ganancias sujeto a los criterios de una sociedad global y consumista.

La educación, lo hemos dicho también en otras ocasiones, más que un gasto es una inversión. Se invierte para generar conocimientos, formar profesionales y técnicos capaces de enfrentar los desafíos de una globalización en marcha, de un cambio de mentalidad, de un uso diferente de las nuevas tecnologías. La educación con calidad, pertinencia e impacto debe ser integral -los valores humanistas, políticos, éticos no pueden perdersedebe ser contextual para entender el entorno y poder responder con efectividad, ser al menos bilingüe, incluir el uso de la informática y de la Internet como forma de comunicación, pero además debe ser entretenida, seductora, lúdico-creativa y con imaginación. Tiene que estimular estilos de vida saludables y el cultivo de los distintos talentos artísticos y cotidianos.

Como usted lo afirmó, señor Rector: “...para el ser humano el conocimiento siempre ha sido importante, pero ahora es fundamental. No hay campo de la vida en el que no influya el saber. Sin ciencia propia, sin un sistema de educación superior vigoroso y de calidad, una sociedad se condena a la maquila, a la medianía en el desarrollo". 
Compartimos el principio señalado por usted al recibir el Premio Príncipe de Asturias, cuando afirma que: "resulta indispensable reivindicar el derecho a la educación. Por ello, es necesario insistir y volverlo a hacer. La educación es vía de la superación humana, de la individual y la colectiva. Concebirla como un derecho fundamental es uno de los mayores avances éticos de la historia. Como bien público y social, la educación superior debe ser accesible a todos bajo criterios de calidad y equidad. Por eso, duele que en el mundo de hoy, con sus grandes desarrollos, vivan cerca de 800 millones de personas que no saben siquiera leer y escribir".

Estamos totalmente convencidos, al igual que usted, de que la educación es la única herramienta realmente útil para luchar contra la desesperanza, el atraso, la marginación y la pobreza.

En una propuesta de desarrollo democrático la Universidad pública juega un papel clave: la Universidad forma profesionales capaces de asumir con competencia, ética y destreza las funciones propias que le demanda el mundo laboral, profesionales con la capacidad creativa y crítica que exige este mundo de rápidas transformaciones, complejo y disímil. En esto, la generación de conocimientos juega un papel de gran relevancia. Es por eso que una institución de enseñanza superior sin desarrollo de la investigación y de la acción social no puede llamarse Universidad en el pleno sentido del término. Desde las universidades públicas se da impulso a una investigación básica y a la aplicada que se multiplica creativamente para solucionar numerosos problemas, logrando un mayor impacto social a corto y mediano plazo. Indiscutiblemente somos instituciones que contribuimos con las transformaciones que la sociedad necesita para el logro del bien común.

Las universidades públicas formamos para responder a la producción, al sector productivo, público y privado, al capital productivo que favorezca los servicios colectivos y contribuya con el mejoramiento de la calidad de vida. Nos interesamos en la teoría puesta en práctica, en la imaginación hecha productividad, en la lógica matemática y el pensamiento filosófico como las bases del proceso. Queremos formar personas que puedan encontrar la información y aplicarla. Personas creativas que puedan generar nuevas estrategias para producir y generar conocimientos más y mejor.

Los países desarrollados han señalado y enfatizado en que una de las medidas para salir de la crisis tiene que ver con el incremento en el financiamiento de la educación superior. No existe en la región centroamericana una universidad pública o privada que desarrolle la cantidad y calidad de trabajo académico sustentado en un modelo de desarrollo 
solidario como lo hace la UCR o lo hacemos las universidades públicas. Fortalecer este modelo, ampliarlo, innovarlo es una reto país y un desafío para una región centroamericana que, como lo estamos viviendo en estos momentos, es vulnerable a los desafíos democráticos y económicos.

En un momento de crisis económica como el que vivimos, el país necesita políticas de estado claras que refuercen el acceso y la participación a las universidades y que lleven a declarar universal, gratuita y obligatoria la enseñanza preescolar y la secundaria.

La crisis del sistema financiero capitalista pone a prueba la relación existente entre hacienda pública, educación superior y desarrollo, no sólo en el sentido cuantitativo de las fórmulas y montos de una transferencia digna, estratégica y garante del orden constitucional, sino también en su sentido cualitativo. Hay que profundizar, ampliar y ofrecer una calidad de vida creciente para un mayor número de personas. El desafío que tiene como país es robustecer la educación en todas las regiones, sobre todo en las que cuentan con mayores niveles de inequidad. Como sociedad debemos garantizar un régimen de transferencias que posibiliten en el corto plazo concretar esta estrategia.

Los países del G-8 o la UNESCO han señalado como una de las medidas para salir de la crisis el imperativo de aumentar el financiamiento de la educación superior, sobre todo, en lo que a la investigación e impacto social se refiere. Y debo decirlo sin humildad, no existe en la región centroamericana y más allá, una universidad o un centro especializado que desarrolle la cantidad y calidad de investigación y acción social como lo hace la UCR y, además, bajo la perspectiva de un modelo de desarrollo solidario.

Como lo dijimos antes, debemos luchar hoy más que nunca para que la educación sea una opción para las comunidades, las familias y las personas como bien público social y no como negocio, como oportunidad de movilidad social y no como límite, que sea una estrategia para lograr equidad y quebrar las diferencias... El Estado tiene el compromiso de financiar la educación superior pública adecuadamente, mediante el Fondo Estatal para la Educación Superior (FEES).

La educación es, sin lugar a dudas, el eje y el fundamento del desarrollo, de un desarrollo democrático y libre, inclusivo, justo y equitativo, donde las personas puedan tener acceso y participar, hacer uso de sus derechos y buscar la armonía y la felicidad. Y a pesar de todas nuestras necesidades de desarrollo, ¿no dicen las encuestas que estamos entre los países más educados y felices del mundo? 